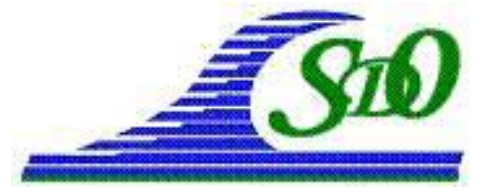

XI ${ }^{\text {èmes }}$ Journées Nationales Génie Côtier - Génie Civil

Les Sables d'Olonne, 22-25 juin 2010

DOI:10.5150/jngcgc.2010.018-Z C Editions Paralia CFL

disponible en ligne - http://www.paralia.fr - available online

\title{
Modélisation numérique du couplage houle - courants pour l'agitation de houle. Comparaison avec des essais en cuve à houle
}

\author{
Bainian ZHANG ${ }^{1}$, Pierre DEBAILLON ${ }^{1}$, Philippe SERGENT ${ }^{1}$ \\ 1. Centre d'Etudes Techniques Maritimes Et Fluviales, \\ 2, boulevard Gambetta, BP 60039, 60321 Compiègne cedex, France. \\ Bainian.zhang@developpement-durable.gouv.fr \\ Pierre.debaillon@developpement-durable.gouv.fr \\ Philippe.sergent@developpement-durable.gouv.fr
}

\section{Résumé :}

La modélisation numérique du couplage houle-courants pour l'agitation de houle est résolue de manière approchée à l'aide d'un modèle aux éléments finis résolvant l'équation de pente douce étendue aux interactions avec les courants. Afin d'évaluer les résultats de ce modèle, une comparaison est effectuée avec des mesures issues d'essais en cuve en houle sur une géométrie schématique contenant un avant-port et l'accès vers un arrière-port. Une première comparaison est réalisée avec des essais de houle sans courants afin d'évaluer les effets de la géométrie et des coefficients de réflexion des ouvrages sur la précision du calcul. En présence de courants entrants la précision du modèle numérique se dégrade. Ce constat n'est pas vrai avec des courants sortants. Cette observation peut s'expliquer par des vitesses de courants entrants plus fortes dans la zone de forte réflexion à l'intérieur de l'avant port. Les interactions houle courants sont en effet moins précises lorsque houles réfléchies et houle incidentes se superposent.

\section{Mots-clés :}

Hydraulique maritime - Agitation de houle - Couplage houle-courants - Modèle de Berkhoff - Equation de pente douce

\section{Introduction}

L'équation de pente douce (BERKHOFF, 1972) est généralement utilisée pour modéliser l'agitation portuaire. Cette équation décrit l'effet combiné de la réfraction et de la diffraction bathymétrique sur la propagation de la houle. Les courants peuvent avoir un effet significatif sur la pénétration des vagues dans les ports, en particulier si ceux-ci sont situés dans les estuaires. Un modèle basé sur l'équation de pente douce intégrant l'effet des courants (KIRBY, 1984), a été résolu par la méthode des éléments finis (KOSTENSE et al., 1988). La validation des interactions complexes entre les houles et les courants est en général réalisée sur des géométries simples. Nous proposons ici de confronter ce type de modèle sur des essais en cuve à houle 
considérant une géométrie schématique contenant un avant-port et l'accès vers un arrière-port.

\section{Modélisation du couplage houle courants}

Nous introduisons le potentiel de vitesse $\phi_{\vec{x} z}(\vec{x}, z, t)$ pour un écoulement irrotationnel avec les coordonnées horizontales $\vec{x}$, la coordonnée verticale $z$ et le temps $t$.

$$
\phi_{x z}(\vec{x}, z, t)=\frac{\cosh [k(h+z)]}{\cosh k h} \phi(\vec{x}, t)
$$

avec $k$ le nombre d'onde $|\vec{k}|$ et $h$ la profondeur. Pour un écoulement périodique, le potentiel complexe $\psi(\vec{x})$ est introduit avec $\omega$ la pulsation et $j$ le nombre complexe dont le carré est -1 .

$\phi(\vec{x}, t)=\operatorname{Re}\left\{\psi(\vec{x}) e^{-j \omega t}\right\}$

L'équation de pente douce sous l'effet d'un courant $\vec{U}$ s'écrit sans terme de dissipation:

$$
\vec{\nabla} \cdot\left[c c_{g} \vec{\nabla} \psi-\vec{U}(\vec{U} \cdot \vec{\nabla} \psi)\right]+2 j \omega \vec{U} \cdot \vec{\nabla} \psi+\left(k^{2} c c_{g}+\omega^{2}-\omega_{r}^{2}+j \omega \vec{\nabla} \cdot \vec{U}\right) \psi
$$

avec la pulsation relative $\omega_{r}=\omega-\vec{k} \cdot \vec{U}$ et l'équation de dispersion corrigée $\omega_{r}^{2}=g k \tanh k h ; c$ et $c_{g}$ représentent respectivement la célérité de phase $\omega_{r} / k$ et la célérité de groupe $d \omega_{r} / d k$. Comme $\omega_{r}, c$ et $c_{g}$ dépendent de la direction inconnue de $\vec{k}$, l'équation (3) est résolue de manière approchée par itération (KOSTENSE et al., 1988). La procédure est la suivante. La pulsation relative $\omega_{r}$ est prise égale à $\omega$ à la première itération en supposant $\vec{U}=0$. L'équation (3) fournit alors la solution $\psi_{0}$ et le vecteur nombre d'onde $\overrightarrow{k_{0}}$.

$\overrightarrow{k_{0}}=\operatorname{Im}\left(\frac{\nabla \psi_{0}}{\psi_{0}}\right)$

A l'itération suivante, la direction de $\overrightarrow{k_{0}}$ est utilisée pour estimer la direction de $\vec{k}$ dans l'équation de dispersion.

$$
\omega_{r}=\omega-\left(\frac{\overrightarrow{k_{0}}}{k_{0}} k\right) \cdot \vec{U}
$$

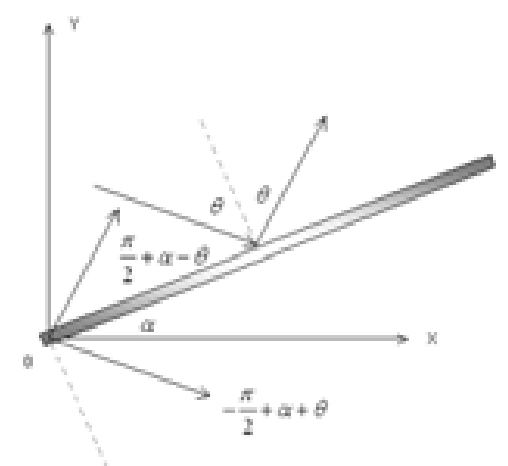

Figure 1. Angles de la houle incidente et réfléchie aux ouvrages . 


\section{XI $I^{\text {èmes }}$ Journées Nationales Génie Côtier - Génie Civil \\ Les Sables d'Olonne, 22-25 juin 2010}

Le processus d'itération (en général moins de cinq itérations) continue jusqu'à ce que la précision attendue soit atteinte. En présence d'une houle incidente et d'une houle réfléchie, il est impossible de prendre en compte parfaitement les directions des deux ondes. Les équations (4) - (5) supposent en effet l'existence d'une direction unique de la houle et donc l'existence d'un angle unique entre la direction de la houle et la direction des courants. La condition aux limites aux frontières réfléchissantes est la suivante :

$\frac{\partial \psi}{\partial n}=j \cos \theta \frac{k_{-\frac{\pi}{2}+\alpha+\theta}-K_{R} k_{\frac{\pi}{2}+\alpha-\theta}}{1+K_{R}} \psi$

avec $K_{R}$ le coefficient de réflexion, $\alpha$ l'angle de la frontière par rapport à l'axe des $x$ et $\theta$ l'angle d'incidence de la houle par rapport à la normale.

L'équation (3) complétée par les conditions aux frontières (6) est résolue par éléments finis et implémentée dans le modèle d'agitation de houle Refonde®.

\section{Modélisation physique en cuve à houle}

L'influence d'un courant sur la pénétration des vagues dans un port et l'agitation portuaire a été étudié en cuve à houle (LUCK et al., 2009) au LNHE à Chatou. L'intérêt principal de la géométrie retenue est d'avoir une zone de diffraction influencée par le courant directement après l'entrée de l'ouvrage. Un schéma de la géométrie et de son implantation dans la cuve est représenté sur la figure $2 \mathrm{a}$. La profondeur est prise constante et égale à $30 \mathrm{~cm}$. Nous disposons de 20 points de mesures de houle dans l'enceinte du port schématique. Ces points sont pris en compte pour le calcul de l'erreur quadratique entre les mesures et le modèle numérique. Nous définissons également les coupes 1 et 2 pour lesquelles des comparaisons entre les mesures et les résultats numériques ont été réalisées. Ces coupes ainsi que les points de mesures sont présentés sur la figure $2 \mathrm{~b}$.

\section{Comparaison houle seule}

La géométrie du domaine peut influer de manière importante le champ de houle calculé dans un port. Par exemple pour des digues à talus, le fait de limiter le domaine au pied ou au sommet des digues modère l'énergie de la houle qui entre dans le port en réduisant plus ou moins la largeur de la passe d'entrée. Disposant de mesures de hauteur de houle sur modèle réduit du LNHE à l'intérieur d'un port schématique à fond plat et délimité par des digues à talus, nous avons ainsi pu évaluer l'influence du choix de la représentation des ouvrages dans la modélisation numérique.

Nous avons ainsi étudié trois représentations pour les ouvrages :

- configuration "sommet" pour laquelle le domaine est limité par une frontière verticale à la hauteur d'eau sur les ouvrages ;

- configuration "milieu" pour laquelle le domaine est limité à la mi hauteur d'eau sur les ouvrages ; 
- configuration "base" pour laquelle le domaine est limité à la base des ouvrages.
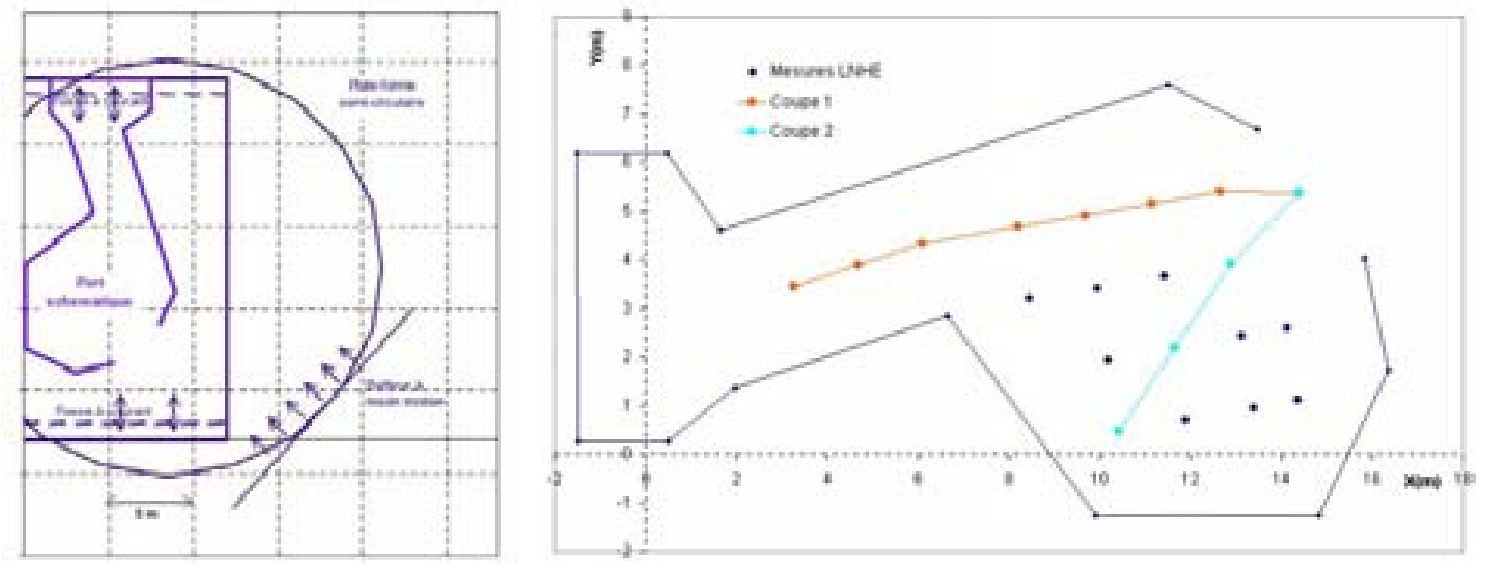

Figure 2. (a) Implantation du port schématique dans la grande cuve d'agitation et (b) les 20 points de mesure et deux coupes comparatives.

\subsection{Sensibilité au coefficient de réflexion et à la représentation des ouvrages}

Pour chaque configuration, nous avons étudié l'influence du coefficient de réflexion affecté aux ouvrages sur l'agitation de houle. Cinq valeurs entre 0,1 et 0,5 ont ainsi été appliquées. En houle régulière, les paramètres de la houle incidente sont les suivantes : hauteur de houle de $4,9 \mathrm{~cm}$, période de $1 \mathrm{~s}$ et direction de $68^{\circ}$ par rapport au Nord. Les maillages associés aux trois configurations ont une densité de 16 nœuds par longueur d'onde. Leurs caractéristiques sont données dans le tableau 1. Le maillage de la configuration "sommet" comporte le plus grand nombre de nœuds en raison de l'épaisseur la plus réduite représentant les ouvrages. Nous avons calculé l'erreur quadratique entre les mesures du LNHE et le modèle numérique pour chaque coefficient de réflexion et chaque configuration. Cette erreur est représentée sur la figure 2.

Tableau 1. Caractéristiques des maillages des trois configurations.

\begin{tabular}{llll}
\hline Configuration & Base & Milieu & Sommet \\
\hline Nombre de næeuds & 94626 & 95896 & 97273 \\
Nombre d'éléments & 187133 & 189699 & 192465 \\
\hline
\end{tabular}

La figure 3 montre que la meilleure configuration est la configuration "milieu ouvrage". La configuration "pied ouvrage" présente une erreur importante pour des coefficients de réflexion moyens. Pour l'ensemble, l'erreur minimale est obtenue avec le coefficient de réflexion le plus faible $(0,1)$ qui est peu réaliste pour un tel ouvrage. En houle aléatoire multidirectionnelle, un spectre de type Pierson-Moskowitz a été imposé sur la frontière circulaire du domaine. Cinq périodes de calcul ont été appliquées $(0.55 \mathrm{~s}, 0.71 \mathrm{~s}, 1.00 \mathrm{~s}$, $1.69 \mathrm{~s}, 5.50 \mathrm{~s}$ ) et cinq directions incidentes $48^{\circ}, 58^{\circ}, 68^{\circ}, 78^{\circ}, 88^{\circ}$ par rapport au Nord. 


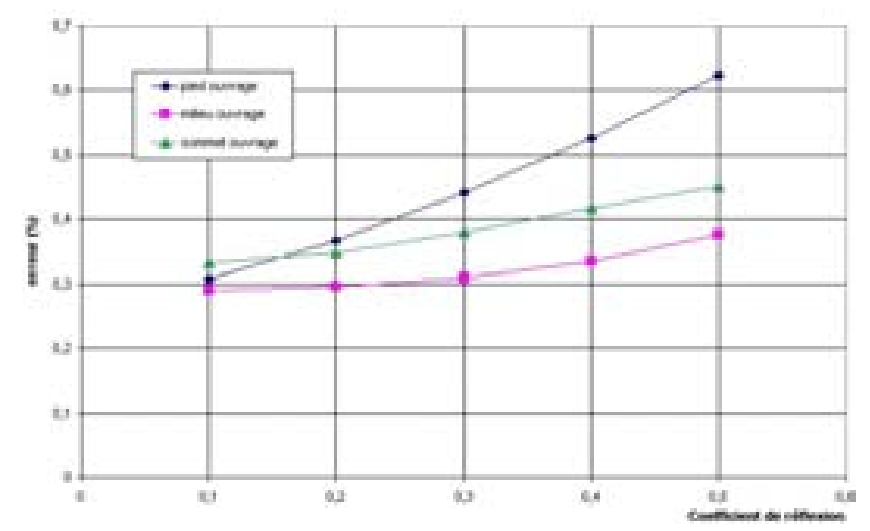

Figure 3. Erreur entre les mesures et le modèle en houle régulière.

Un facteur d'étalement directionnel de 70 a été retenu afin de conserver la direction principale de $68^{\circ}$ prépondérante sur les autres. La figure 4 montre l'évolution de l'erreur quadratique en fonction du coefficient de réflexion pour les trois configurations. On relève une diminution importante de l'erreur pour les trois configurations. On observe également que l'erreur minimale est obtenue par la configuration "milieu" d'ouvrage pour un coefficient de réflexion de 0,4 plus réaliste.

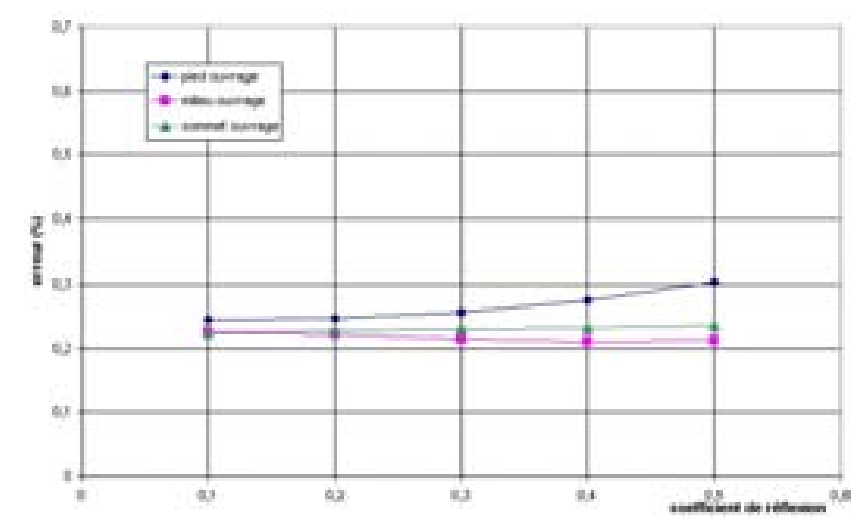

Figure 4. Erreur entre les mesures et le modèle en houle aléatoire multi directionnelle.

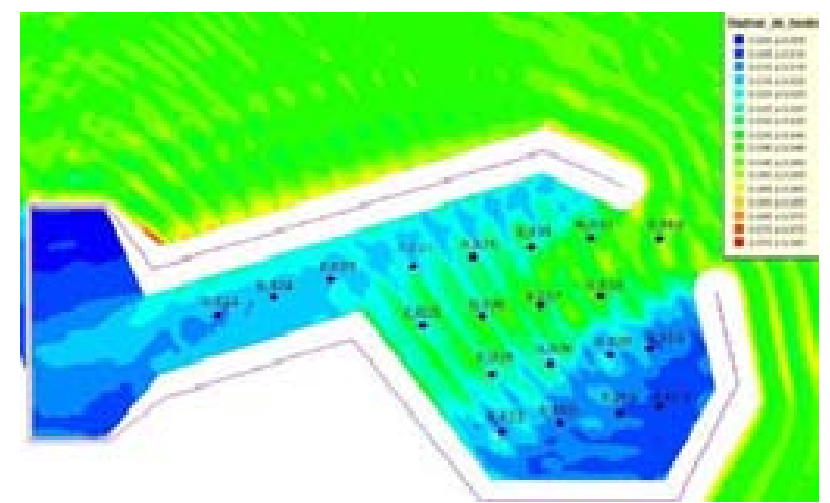

Figure 5. Houle aléatoire multidirectionnelle avec un coefficient de réflexion de 0,4. 


\subsection{Analyse des résultats de la configuration "milieu" pour les ouvrages}

Nous avons retenu la configuration "milieu" pour les ouvrages et superposé le champ de houle calculé par le modèle aux mesures sur la figure 5 avec une houle multidirectionnelle et un coefficient de réflexion de 0,4. La figure 6 montre la relative faible influence du coefficient de réflexion sur le champ de houle. Cependant, le coefficient de réflexion dépend de l'exposition de l'ouvrage à la houle. La figure 6 indique qu'il serait conseillé d'augmenter ce coefficient dans la partie arrière du port abritée de la houle incidente.

\section{Comparaison houle avec courants}

\subsection{Calcul des courants}

Le calcul des courants est effectué à l'aide du logiciel Reflux ${ }^{\circledR}$ qui résout les équations de Saint-Venant par la méthode des éléments finis. Les résultats de deux calculs sont présentés sur les figures $7 \mathrm{a}$ et $7 \mathrm{~b}$ correspondant à des débits de -70 1/s et +70 1/s. Les comparaisons avec les vitesses mesurées (figures $7 \mathrm{a}$ et $7 \mathrm{~b}$ ) donnent des erreurs relatives de $19 \%$ et $20 \%$ respectivement pour les débits sortants et entrants. Une source d'erreur peut encore provenir du choix de la position des frontières verticales sur les ouvrages : ici la configuration nommée «milieu » précédemment. L'expansion des courants dans l'avant-port est plus grande pour le débit entrant que pour le débit sortant.

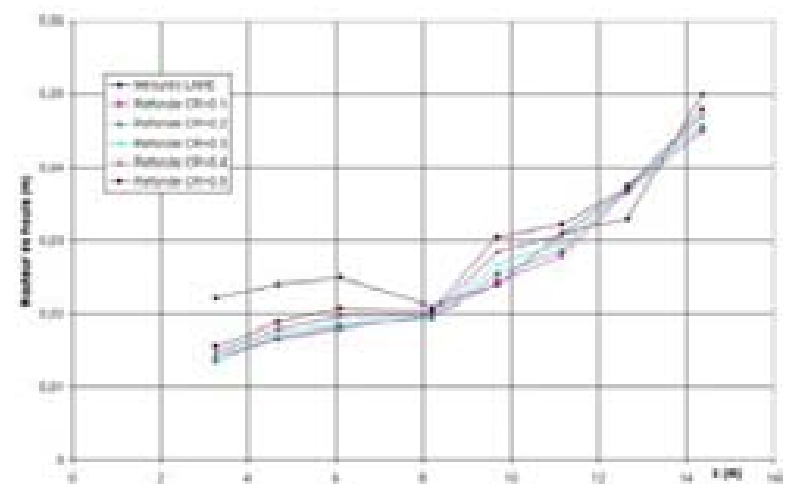

Figure 6. Coupe 1 houle aléatoire multidirectionnelle avec un coef. de réflexion 0,4.
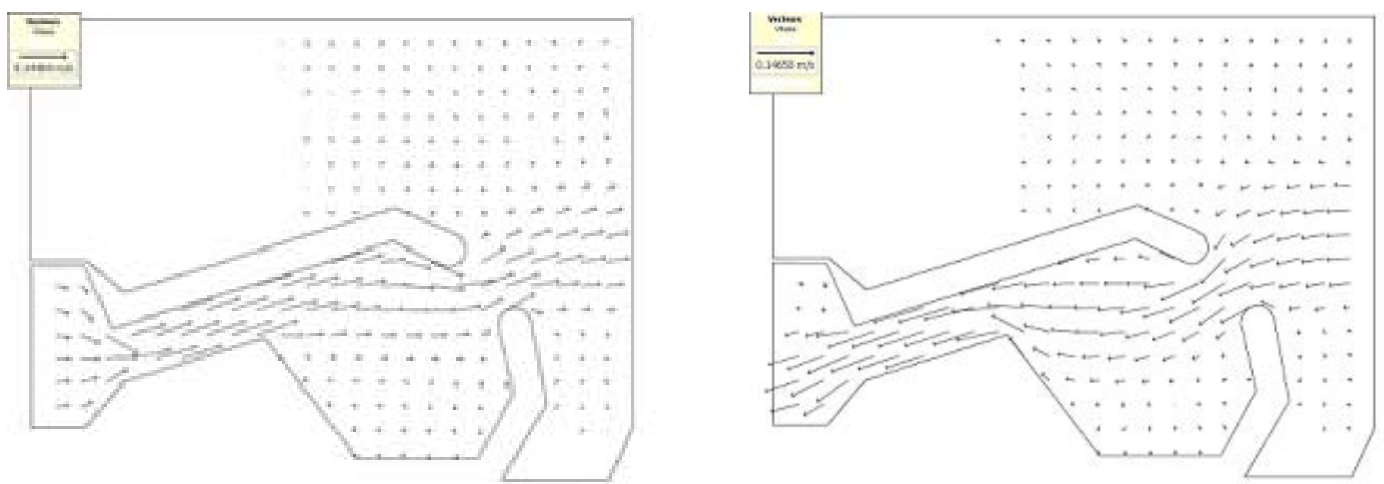

Figure 7. Vitesses des courants pour un débit de $70 \mathrm{l} / \mathrm{s}$. (a) sortant et (b) entrant. 

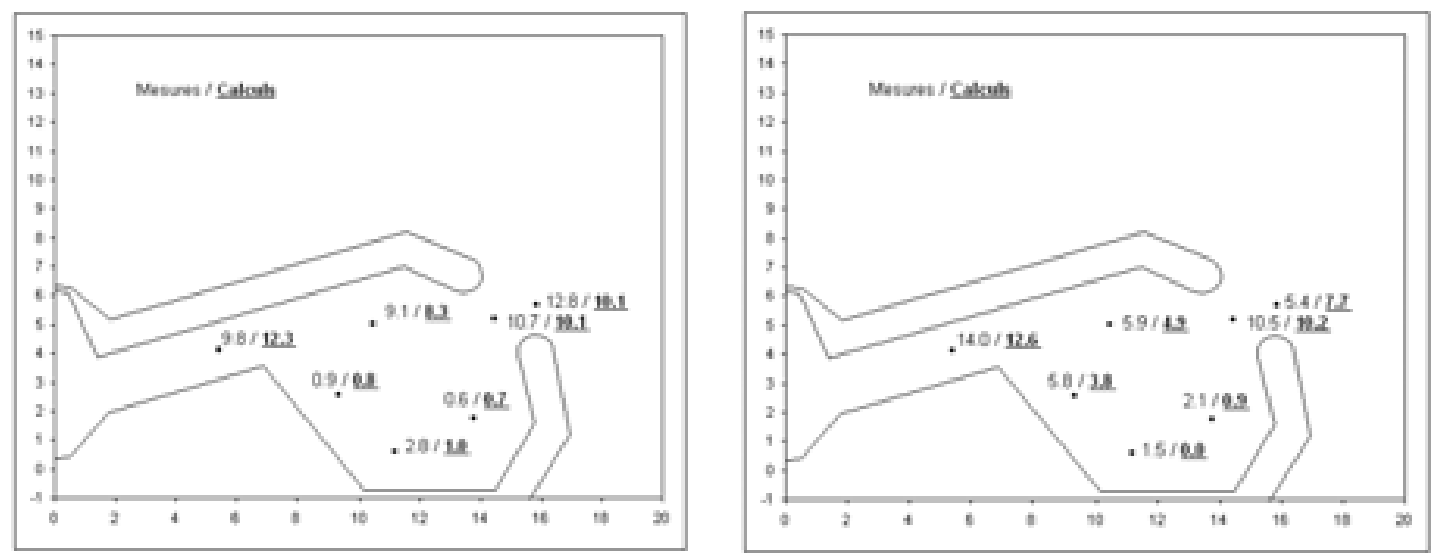

Figure 8. Vitesses des courants mesurées et calculées en $\mathrm{cm} / \mathrm{s}$. (a) pour un débit sortant de $70 \mathrm{l} / \mathrm{s}$ et (b) un débit entrant de $70 \mathrm{l} / \mathrm{s}$.

\subsection{Calcul de la houle}

Des calculs de houle sont effectués pour la configuration «milieu » des ouvrages en houle régulière (coefficient de réflexion égal à 0,2 ) et aléatoire (coefficient de réflexion égal à 0,4 ). Pour le débit entrant, les erreurs relatives atteignent $67 \%$ et $58 \%$ respectivement pour la houle régulière et la houle aléatoire. Pour le débit sortant, les erreurs relatives atteignent $37 \%$ et $28 \%$ respectivement pour la houle régulière et la houle aléatoire. Cette différence s'explique par des vitesses de courants entrants plus fortes dans la zone de forte réflexion à l'intérieur de l'avant port (voir figures 7 et 8). Les interactions houle courants sont en effet moins précises pour les houles réfléchies. La figure 9 présente les hauteurs de houle calculées avec courants. En moyenne sur les 20 points de mesure (cf. figure 1), les hauteurs de houle sont $32 \%$ plus fortes avec le débit sortant que dans la configuration sans courants et seulement $7 \%$ plus faibles avec le débit entrant par rapport à la même configuration. Le tableau 2 présente les hauteurs de houle $(\mathrm{en} \mathrm{cm})$ calculées aux mêmes points. Les différences au-delà $+/-0,5 \mathrm{~cm}(10 \%$ de la hauteur incidente) sont dans les cases grisées.

Tableau 2. Hauteurs $H_{m 0}$ calculées (débit entrant + , sortant - et sans courants 0 ).

\begin{tabular}{lllllllllllllllllllll}
\hline $\boldsymbol{P t}$ & $\mathbf{1}$ & $\mathbf{2}$ & $\mathbf{3}$ & $\mathbf{4}$ & $\mathbf{5}$ & $\mathbf{6}$ & $\mathbf{7}$ & $\mathbf{8}$ & $\mathbf{9}$ & $\mathbf{1 0}$ & $\mathbf{1 1}$ & $\mathbf{1 2}$ & $\mathbf{1 3}$ & $\mathbf{1 4}$ & $\mathbf{1 5}$ & $\mathbf{1 6}$ & $\mathbf{1 7}$ & $\mathbf{1 8}$ & $\mathbf{1 9}$ & $\mathbf{2 0}$ \\
\hline $\boldsymbol{+}$ & 4,7 & 1,4 & 2,3 & 4,0 & 3,9 & 1,4 & 2,5 & 3,7 & 3,1 & 1,7 & 2,5 & 3,0 & 2,6 & 2,3 & 2,1 & 1,9 & 1,8 & 1,7 & 0,8 & 1,6 \\
$\mathbf{0}$ & 5,0 & 1,2 & 2,3 & 4,5 & 4,0 & 0,7 & 2,9 & 4,1 & 3,4 & 1,7 & 2,4 & 3,5 & 3,1 & 2,4 & 2,3 & 2,2 & 2,0 & 1,9 & 0,6 & 1,5 \\
$\mathbf{-}$ & 7,8 & 0,9 & 1,5 & 4,9 & 5,0 & 1,0 & 2,5 & 5,1 & 4,3 & 1,5 & 2,7 & 4,2 & 4,0 & 3,4 & 3,8 & 3,5 & 2,3 & 2,5 & 0,7 & 1,1 \\
\hline
\end{tabular}

\section{Conclusions}

Ce travail de comparaison mesures - calculs sur les interactions houle courants en zone portuaire a montré que les calculs présentent $20 \%$ d'erreur sur les hauteurs de houle ainsi que sur les vitesses des courants, les deux processus étant pris indépendamment. 
En présence conjointe de houle et courants, l'erreur reste du même ordre de grandeur pour des débits sortants. Pour des débits entrants en revanche, cette erreur peut atteindre $60 \%$. Cette différence s'explique par des vitesses de courants entrants plus fortes dans la zone de forte réflexion à l'intérieur de l'avant port. Les interactions houle courants ne sont pas parfaitement modélisées en présence conjointe d'une houle incidente et d'une houle réfléchie. Le modèle est basé en effet sur la donnée d'un angle unique entre la direction de la houle et la direction des courants. Ces imprécisions méritent d'être mieux quantifiées et éventuellement corrigées.

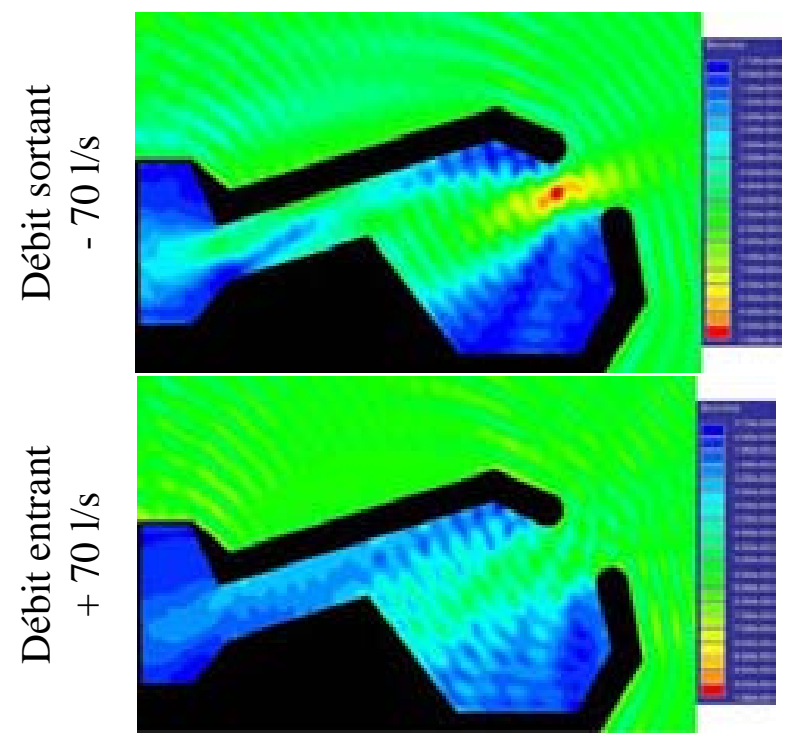

Houle régulière

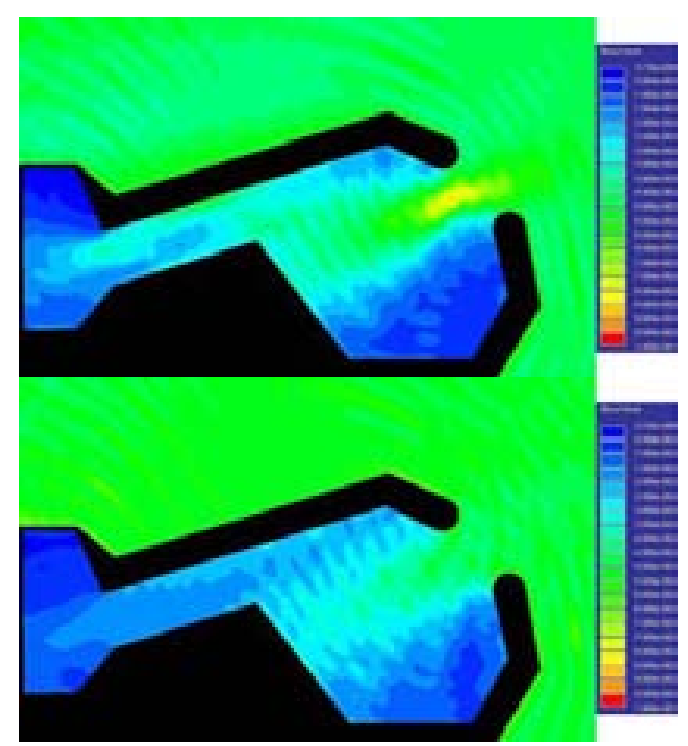

Houle aléatoire

Figure 9. Hauteurs de houle calculées avec courants.

\section{Références bibliographiques}

BERKHOFF J.C.W. (1972). Computation of combined wave refraction-diffraction. Proc. 13th Int. Conf. on Coastal Eng. (ASCE), Vancouver, Canada, pp 471-490.

KIRBY J.T. (1984). A note on linear surface wave-current interaction over slowly varying topography. JGR. Journal of geophysical research, Vol. 89, ${ }^{\circ}$ 1, pp 745-747. doi:10.1029/JC089iC01p00745

KOSTENSE J.K., DINGEMANS M.W., BOSCH VAN DEN P. (1988). Wave-current interaction in harbours. Proc. 21st Int. Conf. on Coastal Eng. (ASCE), New York, Vol. 1, pp 32-46.

LUCK M., MENON J.M., DELISLE J.R. (2009). Etude expérimentale sur les effets du courant sur l'agitation portuaire. Rapport EDF H-P74-2008-01141-FR, 40 p. 